\title{
Neurological asymmetries immediately after unilateral ECT
}

\author{
A. KR IS S, L. D. B L U M H R D T, A. M. H A L L I A Y, A N D \\ R. T. C . P R A T T \\ From the Medical Research Council, Institute of Neurology, and National Hospital for Nervous, \\ Diseases, Queen Square, London
}

SUMMARY Twenty-nine right handed patients were examined neurologically before and immediately after each of 62 unilateral ECTs to the dominant and non-dominant hemispheres. Most convulsions were followed by signs of transitory neurological dysfunction referable to the treated hemisphere. These signs included deep tendon reflex asymmetry, hemiparesis, tactile and visual inattention, and homonymous hemianopia. After treatment to the right hemisphere some patients had left visuospatial neglect, while all patients who had dominant hemisphere ECT were transiently dysphasic. All neurological abnormalities tested resolved within 20 minutes of treatment.

We have previously reported results of separate electrophysiological investigations in which the EEG, flash evoked potential (VEP), and somatosensory evoked potential (SEP) were monitored during the half hour period after unilateral ECT (Kriss et al., 1975, 1977a,b, 1978). Briefly, these studies showed that during the induced seizure the EEG had significantly larger epileptic slow wave activity on the treated side. In the immediate postictal period there was a clear asymmetry with the treated side producing significantly more delta and less alpha and beta activity than the contralateral side. These hemisphere differences, though becoming less marked, persisted beyond the end of the recording. Evoked potential studies showed differing results for the flash VEP and SEP. The VEP was markedly asymmetrical for 15 minutes after the shock with the major positive component $\overline{(P 140)}$ being significantly smaller and later on the treated side. The SEP, however, showed no significant hemisphere differences. In view of these findings we decided to examine patients during the immediate post-ictal period to determine whether there was any clinical evidence of concomitant dysfunction, and to assess the severity and duration of any neurological asymmetries found.

Address for reprint requests: Dr A. Kriss, Medical Research Council, Institute of Neurology, National Hospital, Queen Square, London WCIN 3BG.

Accepted 10 July 1978
Transient neurological abnormalities including reflex changes, aphasia, agnosia, and Gerstmann's syndrome have been reported after conventional bilateral ECT (Hemphill, 1940; Kino, 1943; Juba, 1948; Kalinowsky and Hippius, 1969). Schildge (1952) found that when the stimulating electrodes were placed bioccipitally, six out of 40 patients had transitory blindness usually lasting one to 10 minutes after treatment. Transient neurological abnormalities after unilateral ECT have been referred to anecdotally but do not appear to have been studied systematically. In the introduction to their study of EEG changes after unilateral ECT Bergman et al. (1953) simply state that on recovery of consciousness after treatment “. . . most patients have a transient hemiparesis (Todd's paralysis) . . . some have other signs of dysfunction referrable to the hemisphere stimulated, such as defects in somatic and visual sensation, language dysfunction, and denial of hemiparesis."

Several studies have shown that in the immediate post-ictal period after ECT to the dominant hemisphere, patients have signs of dysphasia which usually resolve within 30 minutes of treatment (Gottlieb and Wilson, 1965; Pratt et al., 1971; Clyma, 1975). More persistent deficits in verbal memory, detected at least an hour after treatment, have also been reported (Cohen et al., 1968; Halliday et al., 1968; Cronin et al., 1970; D'Elia, 1970; Fleminger et al., 1970). Neurological defects in the post-ictal period after non-dominant hemisphere 
ECT do not appear to have been shown but nonverbal/spatial deficits have been reported hours or days after treatment (Cohen et al., 1968; Halliday et al., 1968).

The immediate post-ictal period after unilateral ECT presents a demanding situation in which to examine patients as signs of neurological dysfunction resolve rapidly, usually within 20 minutes of treatment. Because of the transience of the effects, the neurological examination after ECT has to be carried out speedily, yet not superficially, during a period when the patient is responsive but not fully alert. Bearing in mind these limitations tests were chosen which could be executed rapidly, thus allowing serial examinations to be made within a short time. In this way reliance could be placed on any significant changes found and a reasonably accurate assessment of time to recovery of function could be obtained.

\section{Method}

A total of 29 right handed patients (mean age 50.4 years) were examined before and after 62 unilateral ECTs. Forty-seven of the treatments were right sided and 15 left sided. Nine patients had not received unilateral ECT previously while the others had had an average of 3.7 ECTs in their present course of treatment. Fifteen patients in whom cerebral dominance was being established (Pratt et al., 1971; Pratt and Warrington, 1972) were studied after right and left ECT, administered on separate occasions. For a particular patient the same electrical and anaesthetic doses were administered on each occasion.

Deep tendon reflexes, muscle power, tone, and visual fields were assessed before treatment to detect any pre-existing asymmetries. Visual fields were tested by the method of confrontation. Patients were also tested for visual and tactile inattention (Critchley, 1955) by simultaneous bilateral stimulation of homologous areas of their sensory field.

After the physical examination patients were asked to copy or complete a series of figures which included a clock face and a quasi-symmetrical abstract figure.

The shock was an interrupted condenser discharge from a Plexacon Mk II Machine, manufactured by Theratronics Ltd (Strauss and McPhail, 1946). A shock of between 10 and 48 Joules was given with a diphasic mode of discharge. If the shock proved subconvulsive a further stimulus was given. This, however, was rarely necessary. The stimulating electrodes were placed frontocentrally and on the mastoid process of the treated side
(Halliday et al., 1968). A short-acting general anaesthetic, methohexitone sodium (Brietal, 0.88 $\mathrm{mg} / \mathrm{kg}$ body weight), and a muscle relaxant, suxamethonium chloride (Scoline, $1 \mathrm{mg} / \mathrm{kg}$ body weight) were given intravenously. During the immediate post-ictal period patients were oxygenated artificially until spontaneous respiration returned.

In spite of the muscle relaxant, convulsive movements were usually seen after the shock. The location and asymmetry of these were assessed visually. A clock was started at the moment of stimulation, and the duration of the convulsion measured to the last visible twitch or jerk. As soon as the convulsion had ended the post-ictal neurological examination was started. Tendon reflexes were tested repeatedly in the upper (biceps and spinator) and lower limbs (knee jerk), a note being taken of asymmetry and of briskness or depression. The time taken for patients to open their eyes spontaneously and react to instructions was recorded. Visual fields were then examined by confrontation, and the grip strength in each hand compared. Sensation in the upper limbs was tested by light touch or pinprick, first individually to confirm that sensation was present in both limbs, and then simultaneously to test for tactile inattention. If a homonymous field defect was present visual testing was continued until it could not be detected, after which testing for visual inattention was started and continued until its disappearance. If both visual and tactile inattention were present these were tested alternately. When patients appeared to be alert, which did not necessarily mean that their inattention had cleared, they were asked to redraw the test figures.

The 15 patients who received both right and left ECT were also given a verbal test in which they had to name four objects from descriptions (Pratt and Warrington, 1972). As soon as the patient had given his surname correctly the time was noted and the questions posed. If the patient was unable to reply the test was readministered at regular intervals during the recovery period until two or more correct answers were obtained. The time taken to reach this criterion was noted.

\section{Results}

We will present the combined results of all convulsions monitored and include the comparison of signs after right and left ECT where appropriate.

The Table summarises the left/right differences in jerking and twitching observed during the convulsion. Although convulsions were usually bilateral, asymmetries were noted in 57 out of 62 
Table Number of treatments showing significant left/right differences in visually assessed convulsive movements after unilateral ECT

\begin{tabular}{lllll}
\hline $\begin{array}{l}\text { More intense } \\
\text { jerking } \\
\text { throughout on } \\
\text { side } \\
\begin{array}{l}\text { contralateral } \\
\text { to shock }\end{array}\end{array}$ & $\begin{array}{l}\text { Initially more } \\
\text { intense jerking } \\
\text { on contralateral } \\
\text { side but later } \\
\text { more intense } \\
\text { ipsilaterally }\end{array}$ & $\begin{array}{l}\text { Sil } \\
\text { seen }\end{array}$ & $\begin{array}{l}\text { Total } \\
\text { number of } \\
\text { treatments }\end{array}$ \\
\hline $50(81 \%)$ & $7(11 \%)$ & $3(5 \%)$ & $2(3 \%)$ & 62 \\
\hline
\end{tabular}

treatments $(92 \%)$. These asymmetrical features usually consisted of more intense contralateral twitching of the facial musculature, especially at the angle of the mouth and outer canthus of the eye, and more severe jerking of the contralateral arm and hand. Jerking in the legs was not always as asymmetrical as in the upper limbs. In 14 of the 62 fits both feet appeared to show equal involvement. Six treatments produced very localised epileptic movements with only a minimal amount of twitching being seen in the contralateral face or hand (usually index finger or thumb). One convulsion was almost entirely limited to the contralateral neck musculature. In seven treatments, while the convulsive movements initially predominated on the contralateral side they became more pronounced ipsilaterally towards the end of the seizure. One patient, who on separate occasions received right and left ECT, had similar idiosyncratic endings to her convulsions in which abdominal contractions persisted for a short time after twitching in the face and limbs had ceased. Three convulsions appeared to be entirely symmetrical. Two patients showed no visible signs of a convulsion after right ECT.

The mean duration of convulsions was 39.75 seconds (SD 18.45), and there was no significant difference in the duration of seizures evoked by left and right ECT. There was, however, a significant correlation $(r=0.6, \mathrm{P}<0.01)$ between the duration of left and right ECT convulsions in the same patient. As in our previous study (Kriss et al., 1978) a significant correlation was also found between the duration of the convulsion and the time to subsequent eye opening $(r=0.6, \mathrm{P}<0.01)$. It has to be noted, however, that individual anomalies do occur. A few patients appeared consistently to have severe and long convulsions yet awoke more rapidly than expected, while in others the opposite was true. There were no significant correlations between duration of convulsion and shock strength, or the amount of anaesthetic or muscle relaxant used.

Figure 1 shows the percentage of 60 seizures exhibiting asymmetrical neurological signs after

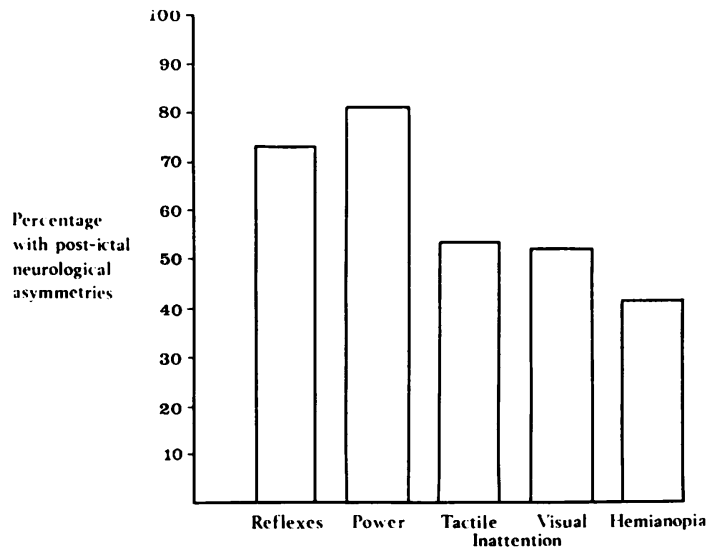

Fig. 1 Percentage of 60 convulsions in 29 patients showing post-ictal neurological asymmetries.

ECT. Figure 2 demonstrates the duration of asymmetries in those cases where the resolution time was determined. The two patients who had no visible convulsion opened their eyes within 2.5 minutes of the shock. As their eye opening recovery times were much quicker than usual (mean $3.4 \mathrm{~min}$, SD $1 \mathrm{~min}$ ) and they awoke with no evidence of neurological dysfunction, we felt in retrospect that the shock had in fact been subconvulsive, and hence these cases were ignored for the purposes of further statistical analysis.

After the convulsion it was usual for the deep tendon reflexes to be depressed for approximately one minute. After this the lower limb jerks returned rapidly, and were usually symmetrical when first tested. In contrast, the upper limb reflexes (biceps and supinator jerks) usually showed an

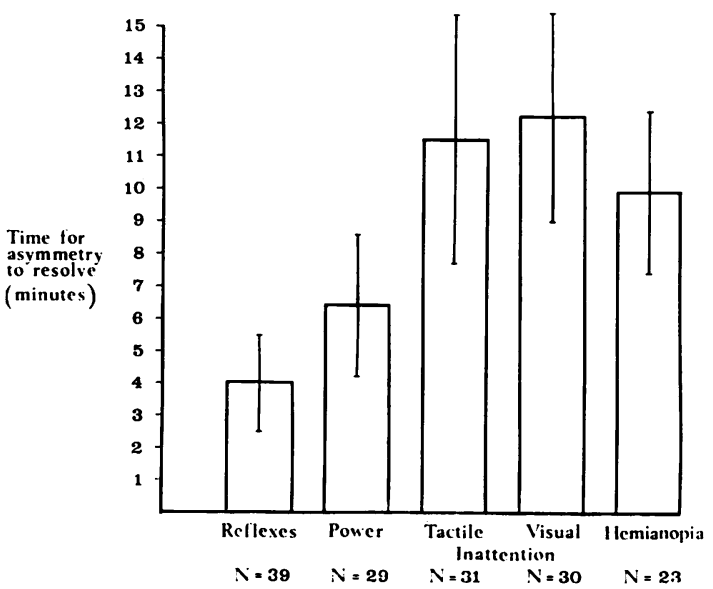

Fig. 2 Time taken after the shock for neurological asymmetries to resolve. 
early asymmetry in which the reflexes ipsilateral to the treated side reappeared first while those on the contralateral side were still depressed. The latter soon returned, and the arm jerks were symmetrical within an average of four minutes after the shock. However, anomalies did occur, and in eight out of 60 convulsions $(13 \%)$ the asymmetry was reversed, with the reflexes on the contralateral side being brisker. Cases which did not have right/left reflex differences tended to have convulsions which were shorter (usually less than 30 seconds) or very localised or symmetrical. There were no significant differences in either the incidence or duration of reflex asymmetries when comparing left and right ECTs (see Figs. 3 and 4).

Once patients had recovered consciousness the power of their limbs was tested. After the majority of convulsions ( 49 out of 60 , that is, $81 \%$ ) there was a flaccid paresis contralateral to the treated side. In general this asymmetrical weakness was either much more marked in, or was even limited to, the upper limbs. Although the marked left/ right differences in grip strength appeared to resolve within 6.5 minutes after the shock, a more subtle disturbance in power could still be demonstrated by drift of the outstretched contralateral arm, a sign which persisted for 12-14 minutes.

The assessment of power asymmetries was complicated in many cases by the presence of "motor inattention" involving the contralateral hand. Thus attempts to test grip strength simultaneously by instructing the patients to grip with both hands at the same time resulted in a response only from the ipsilateral hand, the contralateral hand being ignored although previous independent testing had shown that power had returned to the latter. The

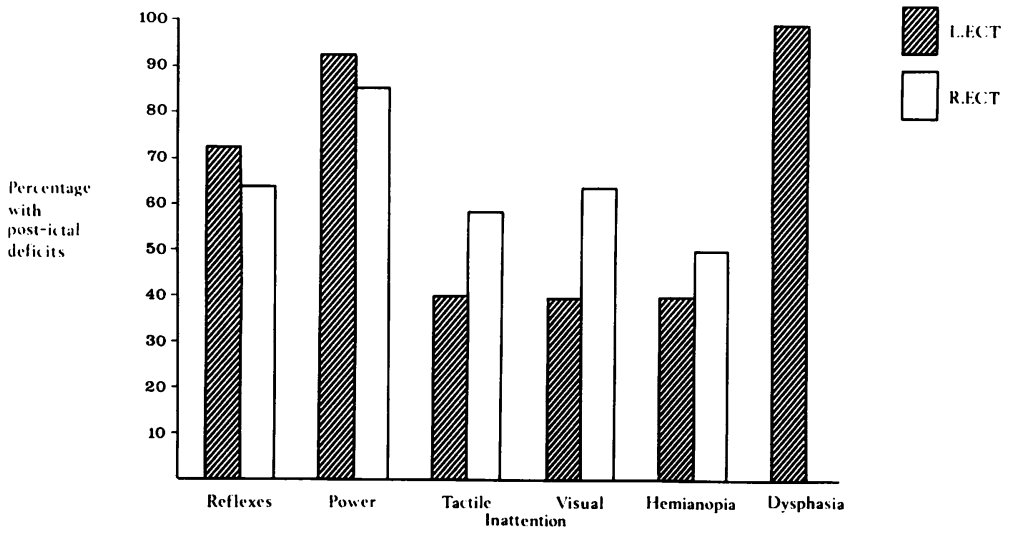

Fig. 3 Percentage of right and left ECT treatments followed by post-ictal neurolog:cal deficits (right ECT $N=14$; left $E C T$ $N=15)$.

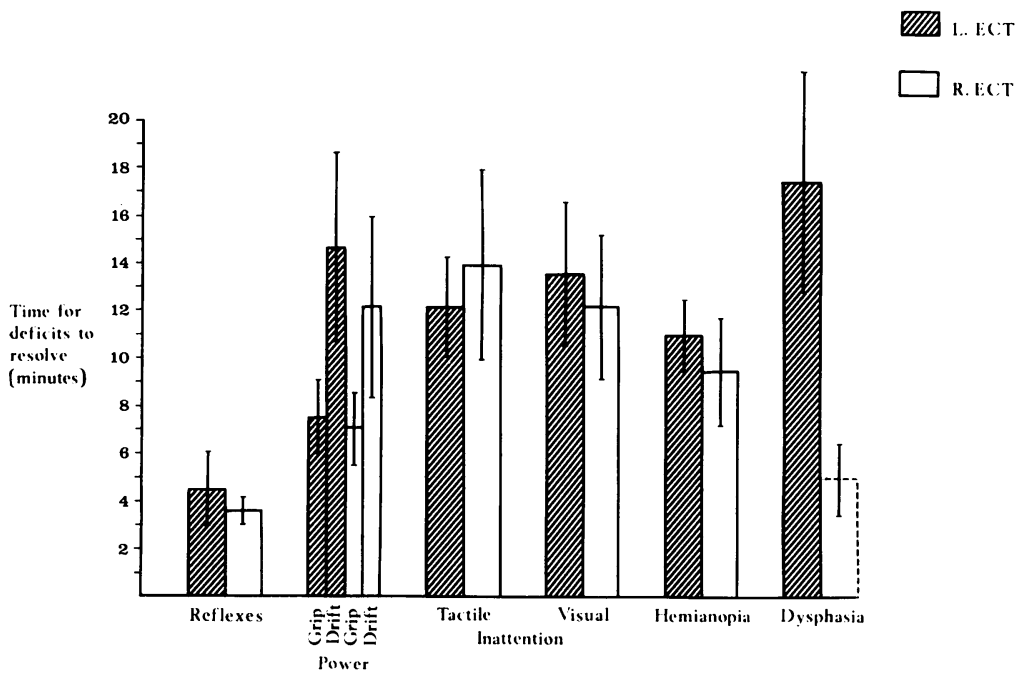

Fig. 4 Comparison of time taken for asymmetries to resolve after right and left ECT. For dysphasia, time taken to name objects from verbal description. 
duration of the grip-power asymmetry was found to be significantly correlated with the duration of the convulsion $(r=0.43, \mathrm{P}<0.01)$.

After 32 out of 60 convulsions (53\%) we found evidence of tactile inattention which, on average, lasted for 11.5 minutes. The duration of this asymmetry showed a just significant correlation with the duration of the convulsion $(r=0.37$, $\mathrm{P}<0.05)$. The incidence and duration of tactile inattention after right and left ECT was not significantly different, although there was a slight numerical trend for it to be more common after right ECT. Three of the eight patients who had left inattention after right ECT had right inattention after left ECT.

Evidence of a homonymous field defect was found after 25 out of $60(41 \%)$ convulsions. These patients had no menace reflex in the half-field contralateral to treatment, but readily blinked on testing in the ipsilateral field. The hemianopia persisted, on average, for just under 10 minutes, and was usually followed by visual inattention on the same side.

In eight out of 60 cases ( $13 \%$ ), visual inattention was not preceded by any detectable hemianopia, at least from the time at which testing first became practicable. On average, visual inattention persisted until 12 minutes after the shock. No significant differences were found in either the incidence or duration of visual inattention after left and right ECT, though again there was a slight but not significant trend for it to be more common after the latter. After 27 out of 60 convulsions $(45 \%)$ tactile and visual inattention were found together, but on six occasions tactile inattention was present without visual inattention, and on five occasions the converse was found.

The test figures were usually drawn satisfactorily
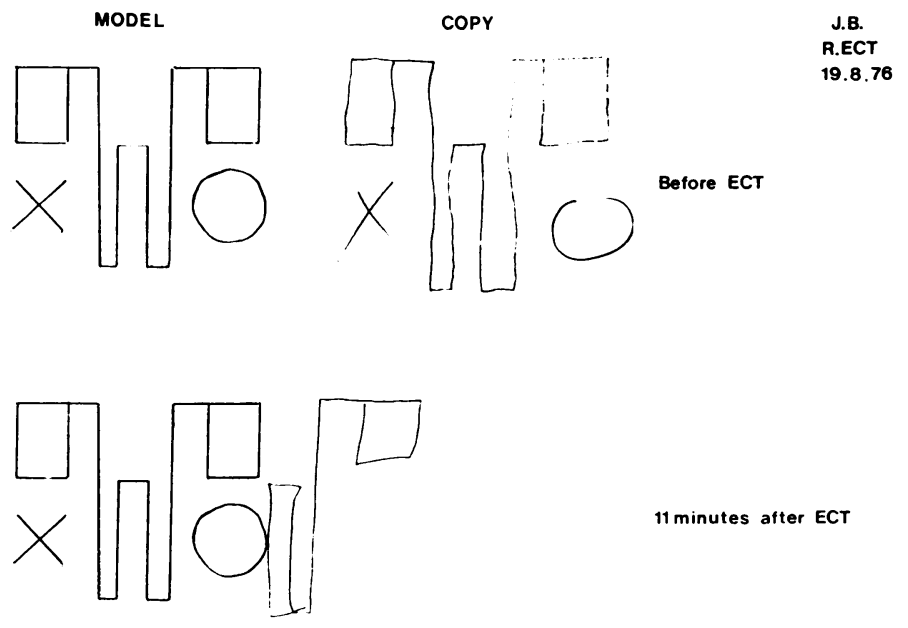

11 minutes after ECT

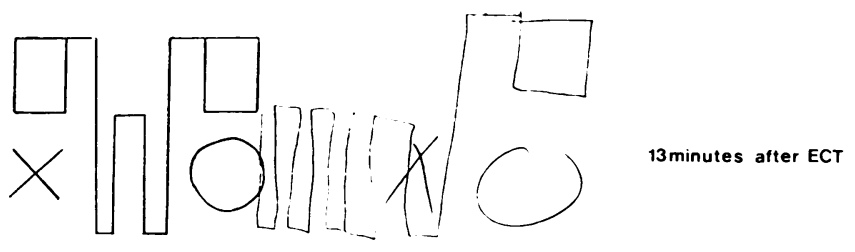

Fig. 5 Abstract figure drawn by a patient who received right ECT. Drawings on the right are the patient's copy of the model. Note neglect of left side 11 and 13 minutes after ECT.
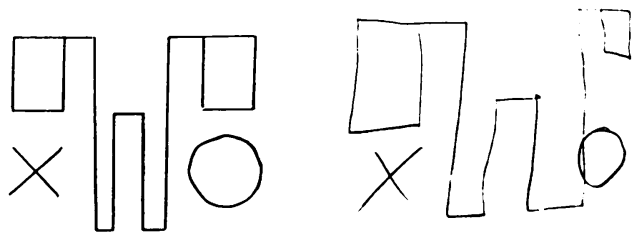

16 minutes after ECT 
after ECT. However, after 15 out of 60 (25\%) convulsions, all right ECTs, errors were made typical of left visuospatial neglect. Figures 5, 6, and 7 show sequences of drawings by different patients who had severe but transient left visuospatial neglect. Errors from other patients were less florid and consisted mainly of greater crowding of numbers on the right side of the clock face and minor omissions on the left side. In many cases, although patients managed to complete figures satisfactorily, they took noticeably longer to draw the left side. Thirteen of the 15 cases who showed evidence of left visuospatial neglect had inattention to the left side of space preceding their draw-
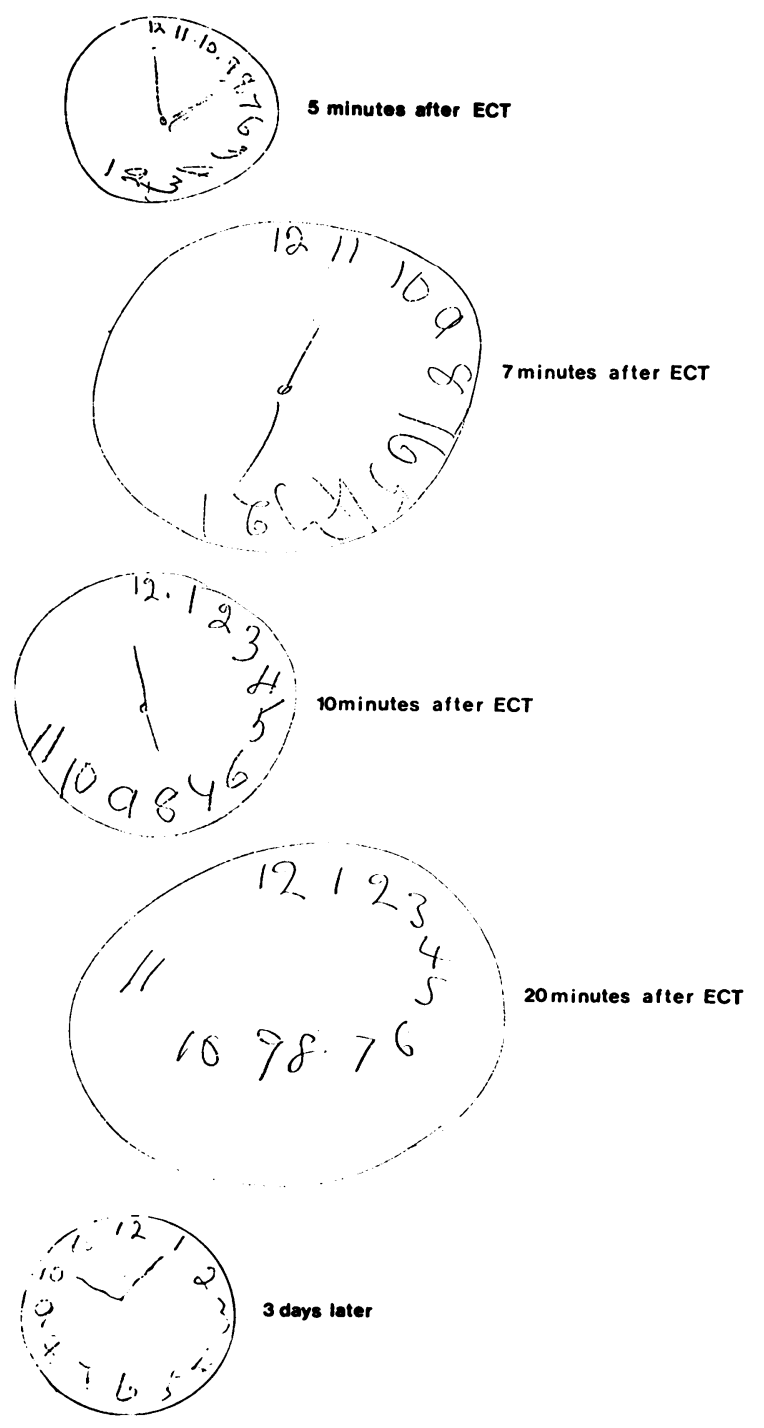

ings. Four of the 15 patients who received both right and left ECT showed evidence of contralateral neglect after right ECT, but no patients had right visuospatial neglect after left ECT.

In general, patients opened their eyes (mean left $\mathrm{ECT}=4.14 \mathrm{~min}$, right $\mathrm{ECT}=3.57 \mathrm{~min}$ ) and gave their surnames (mean left $E C T=9.3 \mathrm{~min}$, right $\mathrm{ECT}=4.8 \mathrm{~min}$ ) significantly faster after right ECT $(P<0.01)$. The naming task was administered only to the group of patients receiving both right and left ECT. The mean time after the shock to answer two or more questions was significantly $(P<0.01)$ shorter after right $(5 \mathrm{~min})$ than after left ECT (17 min). Because of the longer time

F.W.

R.ECT

22.7.78
Fig. 6 Clock faces drawn by another patient after right ECT. Note neglect of left side and reversal in the sequence of numbers in drawings at five and seven minutes after ECT. 

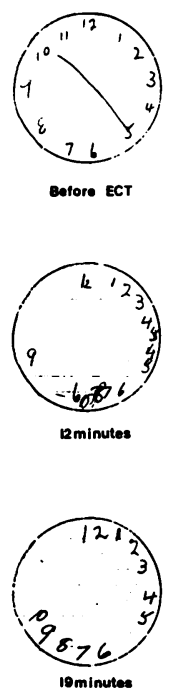
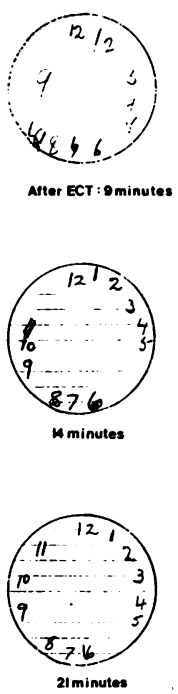
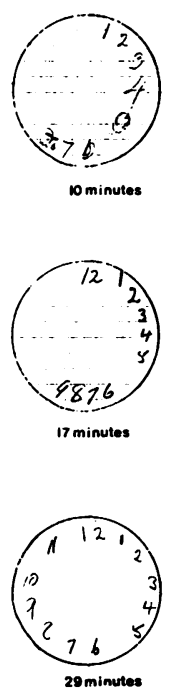

Fig. 7 Longer sequence of clock faces by a third patient also after right ECT. Note neglect of left side and crowding of digits on the right. taken for patients to become responsive after dominant hemisphere ECT, the drawing test was administered at a much later time (mean $14 \mathrm{~min}$ after shock) compared to non-dominant hemisphere treatment (mean 7 min after shock).

Figure 8 shows the number of convulsions of different duration and the incidence of higher neurological dysfunction in each group. The hatched areas represent the proportion of convulsions associated with post-ictal hemianopia or inattention or both. There was a significant trend $(P<0.01)$ for convulsions lasting longer than 30 seconds to be followed by dysfunction.

\section{Discussion}

The results confirm the anecdotal report of
Bergman et al. (1953) that after unilateral ECT there are signs of neurological dysfunction, referable to the treated hemisphere. In the present study we found that most of the induced convulsions, although bilateral, were asymmetrical and were followed by left/right differences in reflex activity and motor power. After approximately half the treatments there were also other signs of lateralised neurological dysfunction including hemianopia, inattention, dysphasia, and visuospatial neglect. All these signs were transient and had usually cleared by about 20 minutes after the shock. It is somewhat surprising that, despite the relatively common use of unilateral ECT, these neurological asymmetries have not been more widely reported before. This may be because they are easily missed, both because of their transitory

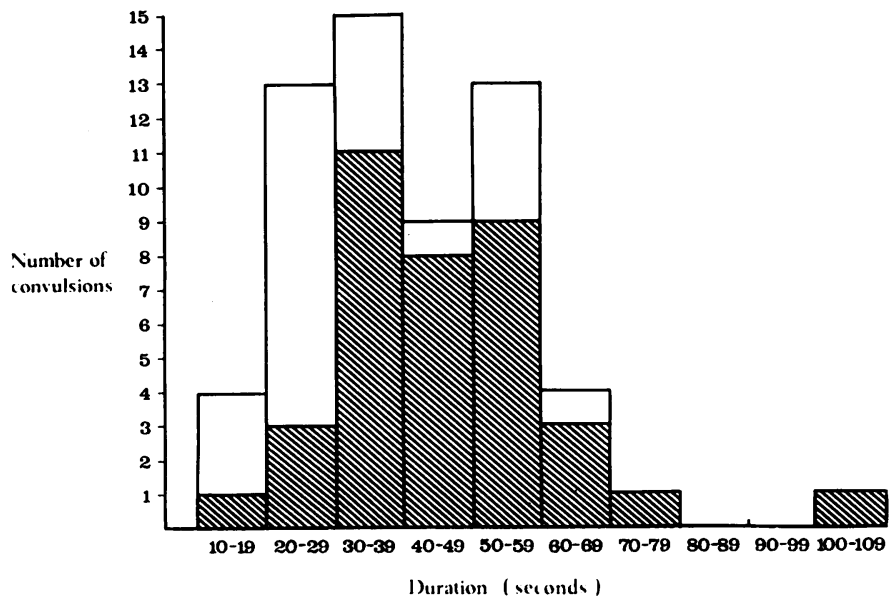

Fig. 8 Relationship of convulsion duration ( 60 convulsions) and incidence of neurological deficits. Hatched areas denote convulsions followed by inattention or hemianopia or both. 
character and because they occur at a time when it is common practice to allow patients to recover undisturbed in a separate room.

These neurological sequelae of unilateral ECT indicate that certainly part, and perhaps all, of the treated hemisphere is functionally depressed for the period immediately after the seizure. Indeed, the depressed reflex activity and flaccid paresis usually observed contralaterally resemble the motor signs after acute unilateral lesions of the motor cortex (Todd, 1854). In a minority of the present cases this asymmetry was reversed or reflexes were symmetrical. Reflex changes have been reported previously after bilateral ECT but with symmetrical reflex exaggeration being more usual (Kino, 1943).

In the present study, transient hemiparesis was the most common sign occurring after unilateral ECT and normally persisted for less than 15 minutes. This duration is, therefore, much shorter than the Todd's paresis seen in epileptics after spontaneous focal (Meyer and Portnoy, 1959) or unilateral seizures (Gastaut and Broughton, 1972).

The additional signs of visual dysfunction indicate that the effects of the treatment were not confined to the area of cortex underlying the stimulating electrodes. A point of interest was that the duration of the visual signs appeared to parallel closely the duration of asymmetries of the flash VEP (Kriss et al., 1977b). In order to elucidate the exact relationship between the VEP and visual defects, it would be necessary to assess simultaneously the neurological and electrophysiological changes in a further study. An interesting incidental observation, in the present results, which may be more than mere coincidence, is that the duration of the hemianopia and the VEP amplitude asymmetry were similar, while the more persistent visual inattention lasted for about the same length of time as the latency asymmetry.

Comparison of the effects of right and left ECT confirmed that the hemisphere on the treated side was the more profoundly affected by the treatment. The defects in higher neurological function resembled the deficits found in lateralised cerebral lesions, with verbal and spatial defects associated with dominant and non-dominant hemisphere lesions respectively (Reitan, 1955; McFie, 1960; Piercy, 1964; Hécaen, 1969). In the present study patients were all right handed. ECT to the left side invariably resulted in gross but transient language dysfunction, as judged by their naming disability. This finding agrees with that of other studies which have assessed verbal performance after unilateral ECT and found it to be worse after treatment to the dominant hemisphere (Gott- lieb and Wilson, 1965; Halliday et al., 1968; Fleminger et al., 1970; Pratt et al., 1971; Clyma, 1975). As all our patients showed signs of dysphasia immediately after left ECT whereas other studies in which testing has taken place up to 20 minutes after the shock have shown a lower percentage, it would seem, as predicted by Pratt et al. (1971), that testing as soon as the patient becomes responsive to questioning may be more sensitive than testing at a later stage.

After right ECT, patients opened their eyes sooner and were significantly quicker in responding. However, after about a third of the nondominant hemisphere treatments patients showed evidence of left spatial neglect when copying figures. In the few cases where neglect was severe its recovery could be followed closely over a short period of time. Recovery is a very unusual feature in other clinical conditions where visuospatial neglect is found (Campbell and Oxbury, 1976). Since only a minority of patients showed signs of left spatial neglect, this test cannot reliably be used for establishing which is the non-dominant hemisphere. Unfortunately, no meaningful comparison can be made of the incidence of neglect after left and right ECT because the slower recovery and more persistent confusion after dominant hemisphere ECT meant that the test could not be administered until a later stage.

The findings of longer time to eye opening and slower responsiveness after left ECT are similar to the trends reported by Halliday et al. (1968), Pratt et al. (1971, 1972), and Clyma (1975), and suggest that the dominant hemisphere has an important role in the maintenance and manifestation of consciousness. Similar conclusions were reached by Serafitinides et al. (1964) based on their studies in which unilateral carotid injections of amylobarbitone were used to depress hemisphere function. These authors reported that consciousness was usually lost after the dominant hemisphere had been injected but was uncommon and transitory when it was administered to the non-dominant side.

In the present study, we noted that the patients had an apparent neglect or unawareness for their neurological disorders. This appeared to be profound and striking after right sided treatment, when the patients were alert and co-operative but apparently unaware, for example, of their severe monoparesis. It is not clear whether there was a comparable defect after dominant hemisphere treatment which was being masked by the greater confusion but it is of interest to remember that clinically anosognosia is particularly associated with non-dominant hemisphere lesions (Brain, 1941, 1962). 
Although there was a general trend for convulsions in the same patient to be of similar duration on different occasions, as shown by the significant correlation between seizure duration after left and right ECT in those patients receiving both, it was noted that there was some variability between treatment sessions for a given patient, particularly in the intensity and bodily distribution of convulsive movements. Furthermore, at times a particular shock intensity failed to evoke a fit where on several previous occasions it proved reliably convulsive. Similar fluctuations in an individual's seizure threshold have been noted in drug-induced convulsive therapy (Kalinowsky and Hippius, 1969). It is not clear why variations in threshold and type of seizure occur, but factors which may play a part include small changes in the position of the stimulating electrodes and in the underlying tissue resistance affecting the amount of current delivered to the brain, the amount of oxygen given before the shock, the time between anaesthetic and shock and, finally, periodic and nonperiodic variations of biochemical and constitutional factors which result in alterations of the brain's susceptibility to epileptic seizures.

Convulsions lasting longer than 30 seconds showed a significant association with the incidence and duration of neurological signs. It appears that it is the character (duration and probably intensity) of the induced seizure which mainly determines post-ictal effects rather than other treatment parameters such as the size of shock or amounts of anaesthetic or muscle relaxant. Ottosson (1960) also stressed the importance of the ECT seizure, especially in determining the therapeutic response and the post-ictal EEG changes. Meyer and Portnoy (1959) studied post-epileptic paralysis in patients with epilepsy due to a variety of causes and in animals after experimental unilateral occlusion of the middle cerebral artery. In chronic experiments on monkeys, it was found that both unilateral and generalised seizures induced by electrical stimulation resulted in transient paralysis in the limbs contralateral to the ischaemic hemisphere. The paralysis was of the flaccid type with absence or depression of tendon jerks. Its duration varied directly with the duration of the seizure. Seizures lasting 45 seconds to one minute tended to produce paralysis lasting three to five minutes. More prolonged seizures resulted in hemiplegia with facial weakness, hemianopia, and loss of response to painful stimuli on the paralysed side. Recovery occurred rapidly, usually over a matter of minutes with return of normal reflexes and tone to the involved side. During post-ictal hemiplegia the threshold voltage required to produce movement by stimulation of the ischaemic hemisphere was found to be five times greater than that required to produce movement by the intact hemisphere.

The similarity in the type and duration of the effects described by Meyer and Portnoy (1959) and those seen in the present study are striking and suggest the possibility that similar pathophysiological mechanisms may be operating after unilateral ECT. Meyer and Portnoy also observed pial circulation through a skull window using a dissecting microscope, and found arterioles to dilate during the seizure with maximum dilatation one to two minutes after its onset and persisting during postictal paralysis. In spite of the increased arteriolar flow the venous circulation appeared cyanotic. In acute experiments on cats, cortical EEG, blood flow, $\mathrm{pH}$ and oxygen tension were concurrently monitored. It was shown that post-epileptic paralysis appeared to be caused by cortical hypoxia resulting from metabolic demand outstripping supply. In a further investigation, Meyer et al. (1966) concluded that post-epileptic paralysis appeared to be initiated by cerebral anoxia but was maintained by cerebral acidosis.

The present study has shown that unilateral ECT has a profound effect on the brain, and that the treated hemisphere is the more severely affected. It would be valuable in further studies to investigate the relationship of the type and duration of the seizure not only to neurological and electrophysiological sequelae, but also to the therapeutic response. More speculatively, it would seem valuable to explore the possibility of combining such a study with an investigation of regional cerebral blood flow.

\section{References}

Bergman, P. S., Impastato, D. J., Berg, S., and Feinstein, R. (1953). Electroencephalographic changes following electrically induced focal seizures. Confinia Neurologica, 13, 271-277.

Brain, W. R. (1941). Visual disorientation with special reference to lesions of the right cerebral hemisphere. Brain, 64, 244-272.

Brain, Lord. (1962). Diseases of the Nervous System, Sixth Edition. Oxford University Press: London.

Campbell, D. C., and Oxbury, J. M. (1976). Recovery from unilateral visuo-spatial neglect? Cortex, 12, 303-312.

Clyma, E. A. (1975). Unilateral electroconvulsive therapy: how to determine which hemisphere is dominant. British Journal of Psychiatry, 126, 372379.

Cohen, B., Noblin, C. D., Silverman, A. J., and Penick, S. B. (1968). Functional asymmetry of the human brain. Science, 162, 475-476. 
Critchley, McD. (1955). The Parietal Lobes. Edward Arnold: London.

Cronin, D., Bodley, P., Potts, L., Mather, M. D., Gardner, C. K., and Tobin, J. C. (1970). Unilateral and bilateral ECT: a study of memory disturbance and relief from depression. Journal of Neurology, Neurosurgery, and Psychiatry, 33, 705-713.

D'Elia, G. (1970). Unilateral electroconvulsive therapy. Acta Psychiatrica Scandinavica, Supplement 215.

Fleminger, J., Horne, D., and Nott, P. (1970). Unilateral electroconvulsive therapy and cerebral dominance: effect of right and left sided electrode placement on verbal memory. Journal of Neurology, Neurosurgery, and Psychiatry, 33, 408-411.

Gastaut, H., and Broughton, R. (1972). Epileptic Seizures. Charles C. Thomas: Springfield, Illinois.

Gottlieb, G., and Wilson, I. (1965). Cerebral dominance: temporary disruption of verbal memory by unilateral electroconvulsive shock treatment. Journal of Comparative and Physiological Psychology, 60, 368-372.

Halliday, A. M., Davison, K., Browne, M. W., and Kreeger, L. C. (1968). A comparison of the effects on depression and memory of bilateral ECT and unilateral ECT to the dominant and non-dominant hemispheres. British Journal of Psychiatry, 114, 997-1012.

Hécaen, H. (1969). Aphasic, apraxic and agnosic syndromes in right and left hemisphere lesions. In Handbook of Clinical Neurology, Edited by P. J. Vinken and G. W. Bruyn, volume 4, pp. 291-311. NorthHolland Publishing Company: Amsterdam.

Hemphill, R. E. (1940). Studies in certain pathophysiological and psychological phenomena in convulsive therapy. Journal of Mental Science, 86, 799.

Juba, A. (1948). Über nach Elektroschock auftretende Kortikale Funktionsstörungen (Gerstmann'sche; Syndrom, Gesichts-und Raumagnosien). Schweizer Archiv für Neurologie und Psychiatrie, 61, 217-226.

Kalinowsky, L. B., and Hippius, H. (1969). Pharmacological, Convulsive and other Somatic Treatments in Psychiatry. Grune and Stratton: New York.

Kino, F. F. (1943). Reflex studies in electrical shock procedure. Brain, 66, 152-161.

Kriss, A., Halliday, A. M., and Pratt, R. T. C. (1975). Asymmetries in the flash evoked response following unilateral electroconvulsive therapy. Electroencephalography and Clinical Neurophysiology, 39, 430.

Kriss, A., Halliday, A. M., and Halliday, E. (1977a). The somatosensory evoked response during uni- lateral electroconvulsive therapy (ECT). Electroencephalography and Clinical Neurophysiology, 42, 144.

Kriss, A., Blumhardt, L., Halliday, A. M., Halliday, E., and Pratt, R. T. C. (1977b). Electrophysiological and neurological asymmetries immediately following unilateral ECT. Electroencephalography and Clinical Neurophysiology, 43, 773.

Kriss, A., Halliday, A. M., Halliday, E., and Pratt, R. T. C. (1978). The EEG immediately after unilateral ECT. Acta Psychiatrica Scandinavica. In press.

McFie, J. (1960). Psychological testing in clinical neurology. Journal of Nervous and Mental Diseases, 131, 383-393.

Meyer, J. S., and Portnoy, R. D. (1959). Post-epileptic paralysis. A clinical and experimental study. Brain, 82, 162-185.

Meyer, J. S., Gotoh, F., and Favale, E. (1966). Cerebral metabolism during epileptic seizures in man. Electroencephalography and Clinical Neurophysiology, 21, 10-22.

Ottosson, J. O. (1960). Experimental studies of the mode of action of electroconvulsive therapy. Acta Psychiatrica Scandinavica, 35, Supplement 145.

Piercy, M. (1964). The effects of cerebral lesions on intellectual function: a review of current research trends. British Journal of Psychiatry, 110, 310-352.

Pratt, R. T. C., and Warrington, E. K. (1972). The assessment of cerebral dominance with unilateral ECT. British Journal of Psychiatry, 121, 327-328.

Pratt, R. T. C., Warrington, E. K., and Halliday, A. M. (1971). Unilateral ECT as a test of cerebral dominance with a strategy for treating left-handers. British Journal of Psychiatry, 119, 79-83.

Reitan, R. M. (1955). Certain differential effects of left and right cerebral lesions in human adults. Journal of Comparative and Physiological Psychology, 48, 474-477.

Schildge, E. (1952). Reversible Corticale Blindhe:t bei Occipital Ausgelöstem Elektroschock. Journal of Nervous and Mental Diseases, 116, 828-840.

Serafitinides, E. A., Hoare, R. D., and Driver, M. V. (1964). Intracarotid sodium amylobarbitone and cerebral dominance for speech and consciousness. Brain, 88, 107-130.

Strauss, E. B., and MacPhail, A. (1946). Steep wave electroplexy. Lancet, 2, 896-899.

Todd, R. B. (1854). Clinical Lectures on Paralysis, Disease of the Brain, and Other Affections of the Nervous System. Churchill: London. 